\title{
Article \\ Finger Tapping as a Biomarker to Classify Cognitive Status in 80+-Year-Olds
}

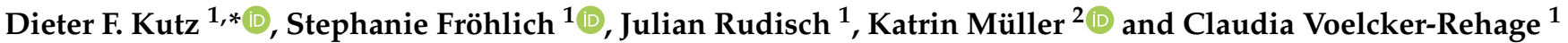 \\ 1 Department of Neuromotor Behavior and Exercise, Institute of Sport and Exercise Sciences, \\ University of Muenster, 48149 Münster, Germany; stephanie.froehlich@uni-muenster.de (S.F.); \\ julian.rudisch@uni-muenster.de (J.R.); claudia.voelcker-rehage@uni-muenster.de (C.V.-R.) \\ 2 Institute of Human Movement Science and Health, Faculty of Behavioural and Social Sciences, \\ Chemnitz University of Technology, 09126 Chemnitz, Germany; katrin.mueller@hsw.tu-chemnitz.de \\ * Correspondence: dieter.kutz@uni-muenster.de
}

check for updates

Citation: Kutz, D.F.; Fröhlich, S.; Rudisch, J.; Müller, K.; Voelcker-Rehage, C. Finger Tapping as a Biomarker to Classify Cognitive Status in 80+-Year-Olds. J. Pers. Med. 2022, 12, 286. https://doi.org/ 10.3390/jpm12020286

Academic Editor: Marco Costanzi

Received: 2 December 2021 Accepted: 11 February 2022 Published: 15 February 2022

Publisher's Note: MDPI stays neutral with regard to jurisdictional claims in published maps and institutional affiliations.

Copyright: () 2022 by the authors Licensee MDPI, Basel, Switzerland. This article is an open access article distributed under the terms and conditions of the Creative Commons Attribution (CC BY) license (https:// creativecommons.org/licenses/by/ $4.0 /)$.

\begin{abstract}
This study examined the association between finger tapping and cognitive function in a group of 225 elderly participants (116 males; age 79-92 years; $M=82.5$; $S D=2.4$ ). Finger tapping was assessed in two conditions: self-selected pace and fast pace. Based on cognitive assessments, including the MoCA and CERA-NP test battery, participants were classified as cognitively healthy individuals (CHI), participants with mild cognitive impairments (MCI), and those with possible MCI (pMCI). Results of the analyses show significant differences between groups, sex and the group $\times$ sex interaction in four parameters for the self-selected pace condition and eight parameters for the fast pace condition. These parameters were used for classification by means of linear discriminant analysis (LDA). The first LDA component showed significant differences between CHI and pMCI and between $\mathrm{CHI}$ and MCI. Furthermore, the second LDA component showed significant differences between $\mathrm{CHI}$ and pMCI as well as between pMCI and MCI. Nevertheless, the algorithm correctly classified only $50 \%$ of participants, regardless of group, suggesting that tapping parameters are only partially useful for classification in early stages of dementia. We discuss these findings in terms of the diadochokinetic nature of finger tapping as associated with the age-related degeneration of cortical and subcortical motor areas.
\end{abstract}

Keywords: aging; cerebellum; classification; cognitive decline; diadochokinesia; motor control; sensory motor performance; time perception

\section{Introduction}

As societies age, more and more people become affected with dementia [1]. In Germany, according to the German Alzheimer Society e.V. [2], the number of people with dementia will have risen to three million by 2050 . In addition to the personal cost, the disease causes substantial economic and social burdens [3]. However, these burdens can be alleviated by early diagnosis of dementia and its pre-stages, as such early detection can allow for more sustainable disease management and optimal health care for affected individuals [1]. It is therefore important to identify people with pre-dementia (e.g., persons with mild cognitive impairments, or MCI) early enough so they can start programs that will help them maintain their personal lifestyle and that will continuously assess the course of the dementia as it progresses.

In many therapeutic areas, diseases and treatments are evaluated using patientreported outcome (PRO) measures (subjective measures), collected, for example, via questionnaires [4]. However, several barriers exist for using PRO measures in cognitive impairment. For example, disease-related disorders can impair memory and cause people to lose insight into how their disease is progressing [4,5]. In these cases, one must rely on the reports of clinicians or information from personal contacts, such as family members $[4,6]$. However, the accuracy of the information provided by family members may be 
suboptimal, as biases may interfere or caregivers may lack knowledge regarding the disease symptoms [4,7]. Therefore, the validity of PRO measurements is limited. Furthermore, the sensitivity of current PRO measures for patients with mild cognitive impaired individuals (MCI) and Alzheimer's disease (AD) patients in the prodromal stage is limited, as they are not specifically designed for these milder conditions [4]. A combination of several neuropsychological tests (e.g., MoCA [8] and CERAD-NP [9]) may improve classification (e.g., $[10,11])$ and additionally identify a transitional stage between cognitively healthy individuals (CHI) and MCI, possible MCI (pMCI) as recently defined as individuals with some signs of cognitive impairment $[1,10]$.

$\mathrm{AD}$ and cognitively healthy individuals have been shown to differ in performance of movement tasks (e.g., finger tapping [12]). Therefore, objective measurements, e.g., by technical systems that measure simple movements, are an alternative to PRO measurements because they are easy to use and inexpensive. For this purpose, researchers use computer-aided measuring systems that measure parameters of the movement by means of a keyboard [13], force sensors [12] or light beams [14]. With these types of devices, studies have shown differences in finger tapping tasks between age-matched healthy subjects and people with AD [12-14], MCI [13,14] and Parkinson's disease [14] in a mean age range of 71-82 years. These differences are mainly related to a slowing of the tapping rhythm and an increase in touch duration as well an increase in the variability of these parameters [12-14]. Such a study on the finger tapping behavior of group pMCI has not been previously conducted. In general, it should be noted that tapping is a diadochokinetic movement consisting of flexion followed by extension of the fingers. The timing of the change in movement is controlled by proprioceptive signals that are triggered when the force sensor is touched [15-17]. The tapping task therefore tests the ability to plan and execute rhythmically oppositely oriented movements. It is shown that the selected tap pace has an influence on the execution [13].

Therefore, in this study we aimed to use tapping parameters to distinguish between participants over 80 years old who were either cognitively healthy individuals (CHI), mild cognitive impaired individuals (MCI) or had possible MCI (pMCI), in two different conditions: as consistently as possible at a self-selected pace or as fast as possible without considering consistency (fast pace). We expected that in addition to reproducing known differences in tapping parameter between CHI and MCI groups [13,14], we would also find differences between pMCI subjects and the other groups. In addition, a recent study has shown for this study group that sex has an effect on force control [18]. It was therefore expected that sex differences in finger tapping parameters would be found. Based on these differences, we then developed a classifier to determine whether a subject belongs to a group.

\section{Materials and Methods}

This study is part of the SENDA study (Sensor-based systems for early detection of dementia, registered in the German Clinical Trials Register under DRKS00013167), which was conducted at Chemnitz University of Technology, Germany. The detailed study protocol was published earlier [1]. Only information relevant to the current research question is described here.

\subsection{Participants}

The SENDA study was advertised by local general practitioners and in newspapers. In total, 244 participants (123 males; age 79-93 years; $\mathrm{M}=82.5$; $\mathrm{SD}=2.5$ ) took part in the study and were recruited from January 2018 to March 2020. Study participation required walking ability, sufficient German language skills, residence in or around Chemnitz, Germany, and a self-organized means of travel to and from the laboratory. Volunteers were excluded before testing if any of the following criteria applied: (1) acute psychological disorder; (2) diagnosis of any neurocognitive or neurological disorder; (3) past traumatic head injury; (4) substance abuse; (5) participation in other clinical studies; (6) a 
physician-directed ban from physical activities; (7) severe restrictions due to cardiovascular, pulmonary, or orthopedic diseases; or (8) failure to reach the minimum required score of 19 during screening with the Montreal Cognitive Assessment [8]. Each participant signed a written informed consent form, and all study proceedings were approved by the Ethics Committee of Chemnitz University of Technology, Germany, Faculty of Behavioral and Social Sciences (V232-17-KM-SENDA-07112017, approved on 19 December 2017). Each participant received 25 EUR compensation for his or her participation at three appointments.

The analysis for this article included 225 participants who took part at the baseline measurement (T1, see [1]). Exclusion from analysis was due to (1) dropout from the study before all needed testing was completed $(n=14)$ or $(2)$ technical issues during the recording $(n=5)$. Due to the participants' old age, many followed a medication regimen $(n=211)$, which most often included medication for high blood pressure $(n=174)$, thrombosis prophylaxis, cholesterol reduction, stomach acid reduction or thyroid function. Demographic characteristics are reported in Table 1.

Table 1. Characteristics of the groups according to cognitive status.

\begin{tabular}{cccc}
\hline & CHI & pMCI & MCI \\
\hline$n$ (in \%) & $79(35)$ & $80(36)$ & $66(29)$ \\
m/f & $35 / 44$ & $43 / 37$ & $38 / 28$ \\
Age in years & $82.0 \pm 0.3$ & $82.5 \pm 0.2$ & $82.9 \pm 0.3$ \\
Education in years & $14.3 \pm 0.4$ & $13.9 \pm 0.4$ & $13.7 \pm 0.4$ \\
MMSE (0-30) & $28.3 \pm 0.2$ & $27.8 \pm 0.2$ & $27.3 \pm 0.2$ \\
MoCA (0-30) & $27.7 \pm 0.1$ & $25.8 \pm 0.2$ & $22.8 \pm 0.2$ \\
\hline
\end{tabular}

Given are means \pm SEM. CHI: cognitively healthy individuals; pMCI: possible MCI; MCI: mild cognitive impaired individuals. For details of the classification, see text. f: female, m: male; MMSE: Mini-Mental State Examination; MoCA: Montreal Cognitive Assessment.

\subsection{Neuropsychological Testing and MCI Classification}

The neuropsychological testing and MCI classification are described in detail elsewhere [10]. Briefly, all participants went through an intensive neuropsychological test battery, which was carried out by trained testing staff at the university lab. The tests included the German version of the MoCA [8] and the German version of the Consortium to Establish a Registry for Alzheimer's Disease Neuropsychological Test Battery [9, CERAD-NP]. The MoCA was used to measure global cognitive functioning and to screen for MCI. The MoCA is the second-most-utilized geriatric cognitive screening tool after the Mini-Mental State Examination (MMSE) and has superior sensitivity to mild cognitive impairments [19]. The CERAD-NP examines the cognitive domains of memory, language, executive functions and visuo-construction. MCI classification was based on the recommendations of The National Institute on Aging and the Alzheimer's Association [20] and in accordance with the criteria proposed by [21]. Cognitive impairments were determined according to performance in MoCA (one sum score) and CERAD-NP (nine separate test scores). The following CERAD-NP scores were used: verbal fluency (number of animals named in $1 \mathrm{~min}$ ), Boston naming test (number of objects correctly identified), phonematic fluency (number of words named with letter "S" in $1 \mathrm{~min}$ ), constructional praxis (number of correctly copied characteristics), word list learning (number of words correctly remembered in third trial), word list recall (savings score), word list recognition (discriminability score), constructional praxis recall (savings score), and trail making test (quotient B/A). We followed a two-step procedure recommended for diagnosis of $\mathrm{MCI}$ in the general population, which states that, first, a screening should be used, and, second, in the case of abnormal findings, in-depth cognitive testing should follow [22]. A MoCA score below 26 points and at least one CERAD-NP test performance at least 1.5 standard deviations below the normative mean (taking into consideration age, sex and education level) resulted in the classification of participants with mild cognitive impairments (MCI). Correspondingly, participants were classified as being cognitively healthy individuals (CHI) if they scored 26 or more points on the MoCA and were also within the normative range 
(no score below -1.5 SD) in all of the CERAD-NP tests [11]. Due to the application of the two-step process, an additional class was defined for participants who showed cognitive impairments only according to one of the two tests. They were categorized as possibly having MCI (pMCI). This group included either participants who had deficits in one specific domain of the CERAD-NP but their overall cognitive functioning was normal according to MoCA, or participants who had no strong impairment in any single domain but had small deficits in different domains adding up to a low MoCA score $(<26)$. Although this group would be considered non-MCI according to [22], as these individuals neither showed abnormal scores in the screening (MoCA > 25) nor exhibited any cognitive impairments in in-depth clinical testing after abnormal testing, we opted to analyze this group separately to have high discriminatory power between $\mathrm{CHI}$ and $\mathrm{MCI}$.

\subsection{Tasks and Recording}

Participants carried out three fine motor tasks [1], including (1) force modulation of a precision grip with the thumb and index finger [18]; (2) tapping with the index finger of the right hand, [based on, 12]; and (3) connecting dots on a touchscreen with a touch pen/tracing (as studied by [23]). Here we report the results of the second motor task, experiment (2).

For the finger tapping tasks, we used one force transducer with a diameter of $29.5 \mathrm{~mm}$, a depth of $8 \mathrm{~mm}$, and a measurement range of $0-22.5 \mathrm{~kg}$ (manufacturer: Measurement Specialties Inc., Hampton, VA, USA; Model: FX-1901-0001-50 L) [18]. Signals were preamplified (using a customized voltage amplifier), digitally converted and sampled at a frequency of $1000 \mathrm{~Hz}$ using a NI-DAQ USB-6002 (National Instruments, Austin, TA, USA). The force transducer was fixed in a self-built wooden board that was placed on the table in front of the participants to prevent any movement of the transducer during the task (see Figure 1a). Experimental procedures, i.e., data acquisition, were programmed using a customized LabView 2015 (National Instruments, Austin, TA, USA) script. The task involved tapping with one's dominant index finger on the force transducer, which participants carried out in two different conditions: as consistently as possible at a selfselected pace (cf., Figure 1b) or as fast as possible without considering consistency (fast pace). Each trial lasted $15 \mathrm{~s}$. In order to reduce the influence of fatigue, the trials were carried out in blocks: the first three trials were in the self-selected pace condition and then two trials were performed for the fast pace condition. Participants received no visual feedback.

\subsection{Data Processing, Parameter Extraction and Statistical Analyses}

Data processing and parameter extraction were performed separately for each trial with a custom-made program in R ([24], ver. 3.6.3). The results were visually inspected and, when necessary, manually corrected. For determining the moment of finger contact with the force transducer, an individual threshold was calculated for each trial using a k-means algorithm with three means. The lowest mean value described the distribution of the noise of the non-contacted force transducer, the highest mean value described the distribution of the force peaks and the remaining mean value described the transition from the noise to the force peaks. The upper $95 \%$ confidence band of the first mean (the noise) was defined as the threshold. For the following analyses, the force curve was low-pass filtered using a second order Butterworth filter (cutoff frequency $100 \mathrm{~Hz}$ ). Individual taps were identified using the filtered force curve. The tap start was defined as the moment when the force curve crossed the threshold after remaining below the threshold for the prior $100 \mathrm{~ms}$. The tap end was the first moment after that when the force curve fell below the threshold (see Figure 1c). Based on the identified taps, the following parameters were extracted:

- tap duration: interval from tap start to tap end;

- tap cycle: interval from a tap start to the following tap start;

- offphase: interval from a tap end to the next tap start, namely the time when the finger is not in contact with the force transducer;

- force peak: force maximum of an individual tap; and 
- time to peak: time from tap start to the moment of the force peak.

Visual inspection of the taps showed that an individual tap could be described by a trapeze (Figure 1c, right tap), having a force increase from tap start onwards (Figure 1c, flexion), reaching a plateau for several milliseconds (Figure 1c, plateau duration) and then followed by a decrease in force until tap end (Figure 1c, extension). To calculate these parameters, the tap was divided into two intervals at time to peak (first interval from tap start to time-to-peak, second from time-to-peak to tap end). For each interval, a two-linear spline model for the force curve over time was calculated [25]. From these calculations, the following parameters were extracted:

- flexion: first force slope in the first interval describing the flexion performance during tapping (Figure 1c, flexion);

- extension: second force slope of the second interval describing the extension performance during tapping (Figure 1c, extension);

- time to plateau: duration from tap start to the break point of the first interval (Figure 1c, right tap). This time describes the duration of the execution of flexion after contact of the finger with the force sensor; and

- plateau duration: duration from the first break point to the second break point (Figure 1c, right tap).

(a)

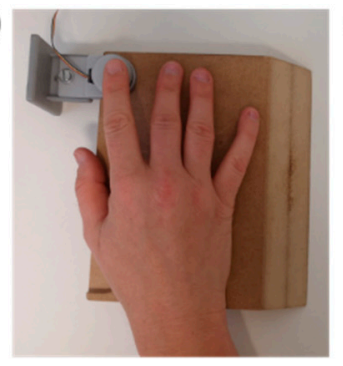

(b)

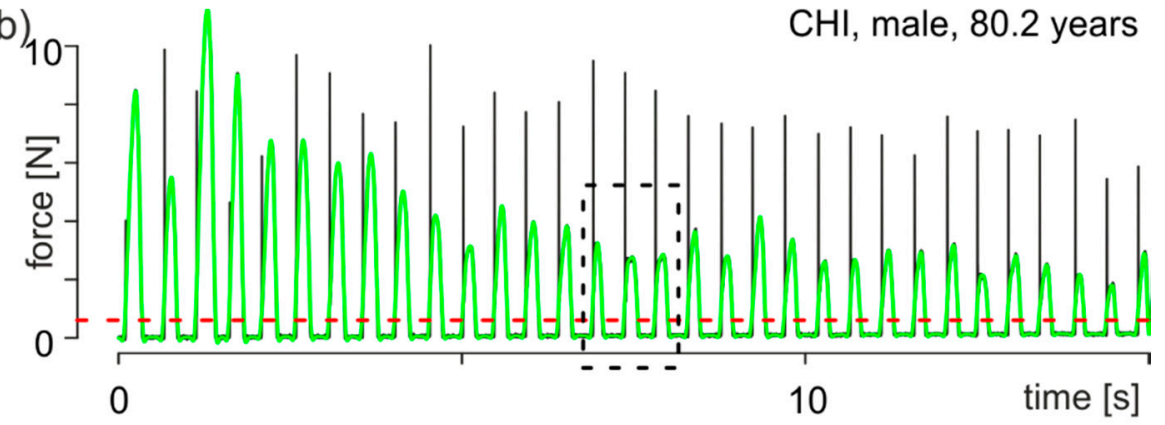

(c) tap-duration

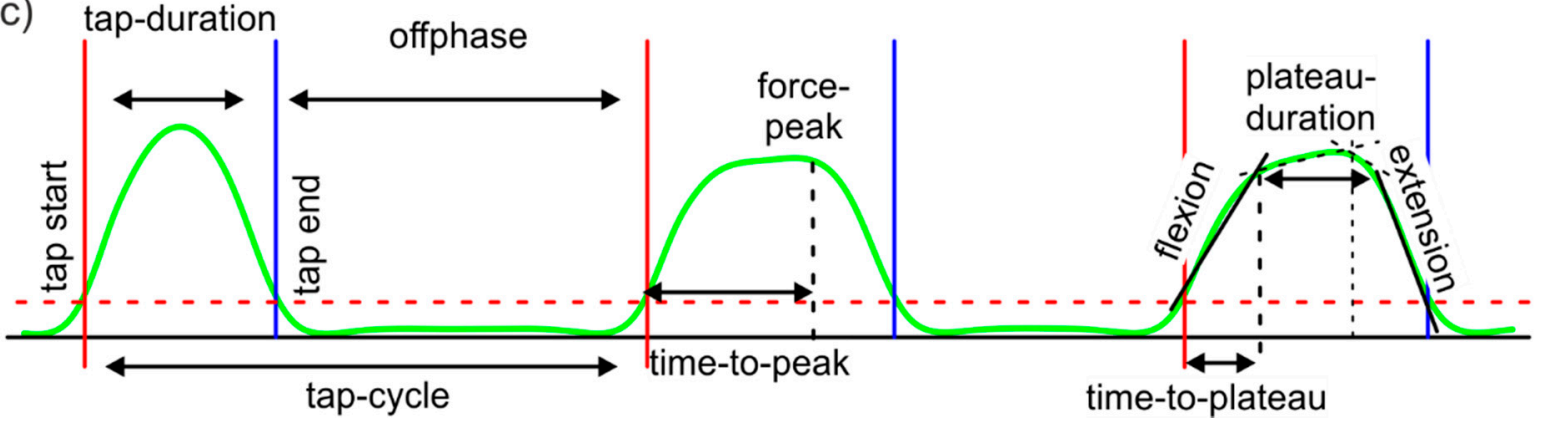

Figure 1. Methods. (a) Experimental setup. (b) Characteristic force curve of tapping. Black line: original recorded force curve; green line: low-pass filtered force curve; red dashed line: force threshold (see text); the dashed rectangle indicates the interval shown in c. (c) Example interval with three taps showing all time and force parameters (see text). Green line: filtered force curve; red vertical solid line: start point of an individual tap; blue vertical solid line: end point of an individual tap; red dashed line: force threshold; black vertical dashed line in the middle tap: time point of the force peak; black vertical dashed lines in the right tap: break points of the two-linear spline models (see text).

Of note, tapping is a diadochokinetic movement consisting of finger flexion followed by finger extension. The time to stop flexion and start extension is controlled by proprioceptive signals [15-17]. Therefore, the mean size of the time to plateau gives information on the planned movement (the shorter the time to plateau, the faster the movement), and its variability gives information on the participant's proprioceptive control at the spinal cord level (the smaller the better). 
All time values were calculated in seconds, the force peak was calculated in Newtons and flexion and extension were calculated in Newtons/second. Since the distributions of a participant's parameters exhibited skewness and kurtosis that could not be fitted by a standard uniform procedure, each participant's individual finger tapping behavior was characterized by two values: first, the size of a parameter using the median of the values and, second, the variability of this parameter using the inter-quartile range (iqr). These values were calculated separately for all parameters in both conditions (self-selected pace and fast pace).

Since the group data were not normally distributed, they were logarithmically transformed for statistical analysis. A mixed ANOVA with a between-subjects factor with two levels (group and sex) and a within-subjects factor (condition) was performed. ANOVA was performed using the $R$ package ez [26]. Effect size $\eta^{2}{ }_{G}$ is given to provide comparability [27]. Post-hoc comparisons were based on Fisher's least significant difference (FLSD) when appropriate. Linear discriminant analysis (LDA) was performed using the R package MASS (version 7.3-53) based on [28]. For LDA, the logarithmized parameters were z-transformed.

\section{Results}

This study was part of the SENDA study [1] and examined the finger tapping behavior of 225 participants over 80 years old who took part at baseline measurement T1 [1]. As described above, the study participants were classified into three groups according to their cognitive performance: cognitively healthy individuals $(\mathrm{CHI}, n=79)$, participants with possible mild cognitive impairments (pMCI, $n=80$ ), and participants with mild cognitive impairments (MCI, $n=66$ ). Overall, 44,813 taps were recorded: 17,724 in the self-selected pace condition and 27,089 in the fast pace condition. In the self-selected pace condition, the groups did not behave differently: CHI performed $86.5 \pm 4.1$ taps on average (mean \pm standard error of the mean (SEM)), pMCI $74.3 \pm 3.4$ taps, and MCI $74.9 \pm 4.6$ taps. In contrast, in the fast pace condition, MCI produced $114 \pm 4.2$ taps, significantly less than CHI (127 \pm 3.2 taps, paired $t$-test with Bonferroni's correction, $p=0.019)$. pMCI (119 \pm 2.9 taps) was not different compared with the other two groups.

\subsection{Statistical Analyses}

\subsubsection{ANOVA}

Group means and standard error of the mean (SEM) of the logarithmized parameter for the two conditions are given in Table 2. For convenience, all group mean values of the tapping parameters have been back-transformed into the respective physical dimensions and are listed in the supplement (Supplementary Table S1). In addition, a sex-specific breakdown of the values can be found in the supplement (Supplementary Table S2 and Supplementary Table S3). ANOVA shows that a total of four (out of 18) parameters in the self-selected pace condition (Table 3 ) and eight (out of 18) in the fast pace condition (Table 4) differed significantly $(p<0.05)$ in group, sex, or the group $\times$ sex interaction. For post-hoc comparison of significant effects, Fisher's least significant difference (FLSD) is given.

Mean values of the parameters (expressed as medians) were different between the groups in both conditions, i.e., the self-selected pace condition and the fast pace condition (Table 2). Post-hoc comparison of tap-cycle_median showed that inside each group, participants tapped faster in the fast pace condition than in the self-selected pace condition $($ FLSD (group + tapping condition $)=0.109)$. In contrast, group comparisons showed that only CHI differed from the other groups in the fast pace condition (FLSD (group) $=0.070$, Table 4). For tap-duration_median, the post-hoc comparison showed that within each group, participants pressed the button for a shorter time during the fast pace condition than during the self-selected pace condition (FLSD (group + tapping condition) $=0.121$ ). In contrast, in the group comparison, only MCI was significantly longer than the others in the fast pace condition (FLSD (group) $=0.070$, Table 4 ). 
Table 2. Logarithmized tapping parameters for both conditions for each group.

\begin{tabular}{|c|c|c|c|c|c|c|}
\hline & \multicolumn{3}{|c|}{ Self-Selected Pace } & \multicolumn{3}{|c|}{ Fast Pace } \\
\hline & CHI $(n=79)$ & pMCI $(n=80)$ & MCI $(n=66)$ & CHI $(n=79)$ & pMCI $(n=80)$ & MCI $(n=65)^{1}$ \\
\hline tap-cycle_median & $-0.593 \pm 0.047$ & $-0.433 \pm 0.045$ & $-0.440 \pm 0.057$ & $-1.442 \pm 0.020$ & $-1.380 \pm 0.026$ & $-1.365 \pm 0.029$ \\
\hline tap-cycle_iqr & $-2.885 \pm 0.081$ & $-2.792 \pm 0.080$ & $-2.703 \pm 0.099$ & $-3.657 \pm 0.066$ & $-3.594 \pm 0.050$ & $-3.449 \pm 0.080$ \\
\hline tap-duration_median & $-1.681 \pm 0.050$ & $-1.510 \pm 0.054$ & $-1.482 \pm 0.066$ & $-2.239 \pm 0.020$ & $-2.203 \pm 0.026$ & $-2.144 \pm 0.029$ \\
\hline tap-duration_iqr & $-3.364 \pm 0.074$ & $-3.173 \pm 0.081$ & $-3.138 \pm 0.089$ & $-3.939 \pm 0.046$ & $-3.914 \pm 0.037$ & $-3.869 \pm 0.053$ \\
\hline offphase_median & $-1.037 \pm 0.051$ & $-0.890 \pm 0.049$ & $-0.915 \pm 0.060$ & $-2.046 \pm 0.025$ & $-1.971 \pm 0.032$ & $-1.983 \pm 0.035$ \\
\hline offphase_iqr & $-2.934 \pm 0.067$ & $-2.836 \pm 0.070$ & $-2.789 \pm 0.083$ & $-3.744 \pm 0.059$ & $-3.751 \pm 0.053$ & $-3.653 \pm 0.075$ \\
\hline force-peak_median & $0.558 \pm 0.109$ & $0.527 \pm 0.104$ & $0.7901 \pm 0.142$ & $0.327 \pm 0.089$ & $0.403 \pm 0.090$ & $0.725 \pm 0.112$ \\
\hline force-peak_iqr & $-0.449 \pm 0.126$ & $-0.594 \pm 0.121$ & $-0.328 \pm 0.123$ & $-0.454 \pm 0.093$ & $-0.420 \pm 0.087$ & $-0.189 \pm 0.119$ \\
\hline time-to-peak_median & $-2.397 \pm 0.054$ & $-2.246 \pm 0.057$ & $-2.195 \pm 0.072$ & $-2.979 \pm 0.019$ & $-2.940 \pm 0.028$ & $-2.884 \pm 0.030$ \\
\hline time-to-peak_iqr & $-3.934 \pm 0.095$ & $-3.710 \pm 0.111$ & $-3.619 \pm 0.112$ & $-4.677 \pm 0.045$ & $-4.631 \pm 0.039$ & $-4.644 \pm 0.057$ \\
\hline flexion_median & $-3.743 \pm 0.082$ & $-3.852 \pm 0.081$ & $-3.676 \pm 0.107$ & $-3.551 \pm 0.080$ & $-3.508 \pm 0.074$ & $-3.228 \pm 0.096$ \\
\hline flexion_iqr & $-4.853 \pm 0.101$ & $-4.935 \pm 0.094$ & $-4.764 \pm 0.104$ & $-4.497 \pm 0.086$ & $-4.497 \pm 0.085$ & $-4.306 \pm 0.104$ \\
\hline extension_median & $-3.735 \pm 0.089$ & $-3.839 \pm 0.086$ & $-3.628 \pm 0.114$ & $-3.650 \pm 0.078$ & $-3.601 \pm 0.074$ & $-3.324 \pm 0.094$ \\
\hline extension_iqr & $-4.766 \pm 0.109$ & $-4.896 \pm 0.090$ & $-4.763 \pm 0.101$ & $-4.624 \pm 0.083$ & $-4.604 \pm 0.076$ & $-4.443 \pm 0.103$ \\
\hline time-to-plateau_median & $-2.722 \pm 0.038$ & $-2.682 \pm 0.040$ & $-2.617 \pm 0.050$ & $-3.053 \pm 0.015$ & $-3.030 \pm 0.021$ & $-2.985 \pm 0.021$ \\
\hline time-to-plateau_iqr & $-4.446 \pm 0.100$ & $-4.448 \pm 0.089$ & $-4.235 \pm 0.108$ & $-4.871 \pm 0.041$ & $-4.855 \pm 0.042$ & $-4.888 \pm 0.057$ \\
\hline plateau-duration_median & $-2.732 \pm 0.076$ & $-2.406 \pm 0.084$ & $-2.408 \pm 0.097$ & $-3.547 \pm 0.025$ & $-3.492 \pm 0.032$ & $-3.446 \pm 0.040$ \\
\hline plateau-duration_iqr & $-3.778 \pm 0.120$ & $-3.457 \pm 0.121$ & $-3.434 \pm 0.133$ & $-5.347 \pm 0.063$ & $-5.278 \pm 0.067$ & $-5.207 \pm 0.083$ \\
\hline
\end{tabular}

Table 3. ANOVA results for the self-selected pace condition. Only parameters with significant differences $(p<0.05)$ in at least one effect are shown. $p$ values are false-discovery-rate-corrected for multiple comparisons.

\begin{tabular}{|c|c|c|c|c|c|c|}
\hline Parameter & Effect & DFn & DFd & $F$ & $p$ & $\eta^{2} G$ \\
\hline \multirow{4}{*}{ force-peak_median } & group & 2 & 219 & 1.22 & 0.30 & 0.01 \\
\hline & sex & 1 & 219 & 6.32 & 0.03 & 0.03 \\
\hline & group $\times$ sex & 2 & 219 & 3.85 & 0.06 & 0.03 \\
\hline & FLSD (sex) & 0.263 & & & & \\
\hline \multirow{4}{*}{ flexion_median } & group & 2 & 219 & 1.04 & 0.35 & 0.01 \\
\hline & sex & 1 & 219 & 7.43 & 0.03 & 0.03 \\
\hline & group $\times$ sex & 2 & 219 & 3.23 & 0.06 & 0.03 \\
\hline & FLSD (sex) & 0.200 & & & & \\
\hline \multirow{4}{*}{ extension_median } & group & 2 & 219 & 1.18 & 0.31 & 0.01 \\
\hline & $\operatorname{sex}^{1}$ & 1 & 219 & 6.81 & 0.03 & 0.03 \\
\hline & group $\times$ sex & 2 & 219 & 3.36 & 0.06 & 0.03 \\
\hline & FLSD (sex) & 0.214 & & & & \\
\hline \multirow{4}{*}{$\begin{array}{c}\text { plateau- } \\
\text { duration_median }\end{array}$} & group & 2 & 219 & 4.49 & 0.03 & 0.04 \\
\hline & sex & 1 & 219 & 1.68 & 0.30 & 0.01 \\
\hline & group $\times$ sex & 2 & 219 & 0.99 & 0.37 & 0.01 \\
\hline & FLSD (group) & 0.238 & & & & \\
\hline
\end{tabular}

DFn: Degree of freedom (nominator); DFd: degree of freedom (denominator); $\eta^{2}{ }_{G}$ : generalized effect size; group CHI, pMCI, MCI; sex: male, female; group $\times$ sex: interaction between group and sex; FLSD: Fisher's least significant difference. 
Table 4. ANOVA results for the fast pace condition. Only parameters with significant differences $(p<0.05)$ in at least one effect are shown. $p$ values are false-discovery-rate-corrected for multiple comparisons.

\begin{tabular}{|c|c|c|c|c|c|c|}
\hline Parameter & Effect & DFn & DFd & $\mathbf{F}$ & $p$ & $\eta^{2} G$ \\
\hline \multirow{6}{*}{ tap-cycle_median } & group & 2 & 218 & 3.41 & 0.03 & 0.03 \\
\hline & sex & 1 & 218 & 6.62 & 0.015 & 0.03 \\
\hline & group $\times$ sex & 2 & 218 & 5.24 & 0.015 & 0.05 \\
\hline & FLSD (group) & 0.070 & & & & \\
\hline & FLSD (sex) & 0.057 & & & & \\
\hline & FLSD $($ group $\times$ sex $)$ & 0.097 & & & & \\
\hline \multirow{4}{*}{ tap-cycle_iqr } & group & 2 & 218 & 3.24 & 0.06 & 0.03 \\
\hline & $\operatorname{sex}$ & 1 & 218 & 7.11 & 0.03 & 0.03 \\
\hline & group $\times$ sex & 2 & 218 & 2.11 & 0.12 & 0.02 \\
\hline & FLSD (sex) & 0.147 & & & & \\
\hline \multirow{5}{*}{ tap-duration_median } & group & 2 & 218 & 3.63 & 0.045 & 0.03 \\
\hline & $\operatorname{sex}$ & 1 & 218 & 0.10 & 0.75 & 0.00 \\
\hline & group $\times$ sex & 2 & 218 & 4.04 & 0.02 & 0.04 \\
\hline & FLSD (group) & 0.070 & & & & \\
\hline & FLSD (group $\times$ sex $)$ & 0.098 & & & & \\
\hline \multirow{5}{*}{ offphase_median } & group & 2 & 218 & 2.69 & 0.07 & 0.02 \\
\hline & $\operatorname{sex}$ & 1 & 218 & 13.4 & $<0.001$ & 0.06 \\
\hline & group $\times$ sex & 2 & 218 & 4.51 & 0.015 & 0.04 \\
\hline & FLSD (sex) & 0.069 & & & & \\
\hline & FLSD $($ group $\times$ sex $)$ & 0.117 & & & & \\
\hline \multirow{4}{*}{ offphase_iqr } & group & 2 & 218 & 1.09 & 0.34 & 0.01 \\
\hline & sex & 1 & 218 & 10.8 & 0.003 & 0.05 \\
\hline & group $\times$ sex & 2 & 218 & 1.25 & 0.34 & 0.01 \\
\hline & FLSD (sex) & 0.137 & & & & \\
\hline \multirow{5}{*}{ force-peak_median } & group & 2 & 218 & 4.04 & 0.02 & 0.04 \\
\hline & sex & 1 & 218 & 4.83 & 0.03 & 0.02 \\
\hline & group $\times$ sex & 2 & 218 & 1.60 & 0.20 & 0.01 \\
\hline & FLSD (group) & 0.267 & & & & \\
\hline & FLSD (sex) & 0.219 & & & & \\
\hline \multirow{5}{*}{ flexion_median } & group & 2 & 218 & 3.72 & 0.045 & 0.03 \\
\hline & $\operatorname{sex}$ & 1 & 218 & 6.34 & 0.03 & 0.03 \\
\hline & group $\times$ sex & 2 & 218 & 1.06 & 0.35 & 0.01 \\
\hline & FLSD (group) & 0.230 & & & & \\
\hline & FLSD (sex) & 0.188 & & & & \\
\hline \multirow{5}{*}{ extension_median } & group & 2 & 218 & 3.78 & 0.03 & 0.03 \\
\hline & $\operatorname{sex}$ & 1 & 218 & 8.10 & 0.015 & 0.04 \\
\hline & group $\times$ sex & 2 & 218 & 1.03 & 0.36 & 0.01 \\
\hline & FLSD (group) & 0.227 & & & & \\
\hline & FLSD (sex) & 0.185 & & & & \\
\hline
\end{tabular}

DFn: Degree of freedom (nominator); DFd: degree of freedom (denominator); $\eta^{2}{ }_{\mathrm{G}}$ : generalized effect size; group CHI, pMCI, MCI; sex: male, female; group $\times$ sex: interaction between group and sex; FLSD: Fisher's least significant difference.

In the fast pace condition, pMCI was significantly different from $\mathrm{CHI}$ for the parameter offphase_median and from MCI for the parameters force-peak_median, flexion_median, and extension_median. Therefore, for motor behavior, pMCI can be considered its own group between $\mathrm{CHI}$ and MCI. In addition, MCI differed significantly from $\mathrm{CHI}$ in the fast pace condition for the parameters tap-cycle_median, tap-cycle_iqr, tap-duration_median, force-peak_median, and flexion_median. In contrast, all significant variability measures showed sex differences (tap-cycle_iqr, offphase_iqr, Table 4).

Individual parameters clearly had small effect sizes, as shown by $\eta G^{2}$. The highest significant value for group was 0.0393 (plateau-duration_median at self-selected pace; Table 3), for sex it was 0.0579 (offphase_median at fast pace; Table 4), and for group $\times$ sex it was 0.046 (tap-cycle_median at fast pace; Table 4). Hence, none of the parameters alone were suitable for assigning individual participants to one of the groups. Instead, classifying 
participants required a combination of parameters with significant effects for group sex, or group $\times$ sex.

\subsubsection{Linear Discriminant Analysis}

Linear discriminant analysis (LDA) is a method of finding a linear combination of features that characterizes two or more classes of parameters. The resulting combination reduces the dimensionality and is used to classify the participants. Because a recent study for this study group showed that sex has an effect on force control [18], LDA was performed not only with parameters of the effect group but also with parameters of the effect of sex and the interaction group $\times$ sex. In total, four parameters of the self-selected pace condition and eight parameters of the fast pace showed significant differences $(p<0.05)$ for the effects of group, sex or the interaction group $\times$ sex. These parameters were analyzed using the R package MASS (see [28]). The LDA showed that the parameters can be combined into two linear combinations, LDA1 and LDA2. LDA1 explains 70\% of the variance and LDA2 30\%. The scales of the parameters are given in Table 5. Note that the suffix _self specifies the parameter of the self-selected pace condition and _fast specifies that of the fast pace condition.

Table 5. LDA scales.

\begin{tabular}{ccc}
\hline Parameter & LDA1 & LDA2 \\
\hline force-peak_median_self & 0.38 & 0.93 \\
flexion_median_self & -0.61 & -0.97 \\
extension_median_self & -0.69 & 0.21 \\
plateau-duration_median_self & 0.50 & -0.69 \\
tap-cycle_median_fast & -0.04 & -4.50 \\
tap-cycle_iqr_fast & 1.01 & 0.31 \\
tap-duration_median_fast & 0.31 & 2.98 \\
offphase_median_fast & 0.17 & 2.45 \\
offphase_iqr_fast & -1.02 & 0.09 \\
force-peak_median_fast & -1.42 & -3.17 \\
flexion_median_fast & 0.91 & 3.27 \\
extension-median_fast & 1.26 & -0.15 \\
\hline
\end{tabular}

The suffix _self specifies the parameter of the self-selected pace condition and _fast specifies that of the fast pace condition.

The distribution of the LDA scales of each group showed a significant difference of the medians among them for LDA-1 (Figure 2a). Post-hoc tests by means of a pairwise Wilcoxon rank-sum test confirmed the group difference for LDA1 between CHI and pMCI $(p<0.001$, Bonferroni corrected) and between CHI and MCI $(p<0.001$, Bonferroni corrected). For LDA-2 (Figure $2 b$ ), there was a difference in medians between $\mathrm{CHI}$ and $\mathrm{pMCI}$ as well as between MCI and pMCI. The post-hoc tests for LDA2 confirmed the significant difference between $\mathrm{pMCI}$ and $\mathrm{MCI}(p<0.05$, Bonferroni corrected $)$ and between $\mathrm{pMCI}$ and $\mathrm{CHI}$ only at a trend level $(p<0.1$, Bonferroni corrected). The same test for $\mathrm{CHI}$ vs. $\mathrm{MCI}$ revealed that for LDA2, the two groups were not significantly different at all ( $p=1$, Bonferroni corrected).

Astonishingly, reclassifying the participants based on the linear discriminant analysis only categorized $50 \%$ of participants into the right class, with the goodness of classification decreasing from $\mathrm{CHI}$ ( 49 of 79 ) to $\mathrm{pMCI}(41$ of 80$)$ to $\mathrm{MCI}$ ( 23 of 65$)$. This is better than the theoretical probability of $1 / 3$, but $50 \%$ were still misclassified (Table 6 ). The sensitivity of this classification for each group was $\mathrm{CHI}=0.62, \mathrm{pMCI}=0.51$, and $\mathrm{MCI}=0.35$; the specificity for each group was $\mathrm{CHI}=0.70, \mathrm{pMCI}=0.69$, and $\mathrm{MCI}=0.86$. Upon further inspecting the distribution of LDA1 and LDA2 over all correctly classified participants and misclassified participants, we found that for LDA1, correctly classified CHI participants were indeed different on this scale relative to correctly classified pMCI and MCI participants (Figure 3a). For LDA2, the difference between correctly classified pMCI participants and correctly classified participants of the other groups was clearly visible (Figure 3b). Importantly, the 
LDA's inability to properly classify participants was related to the broad distribution of misclassified participants on both LDA1 and LDA2 scales (gray histograms in Figure 3a,b).

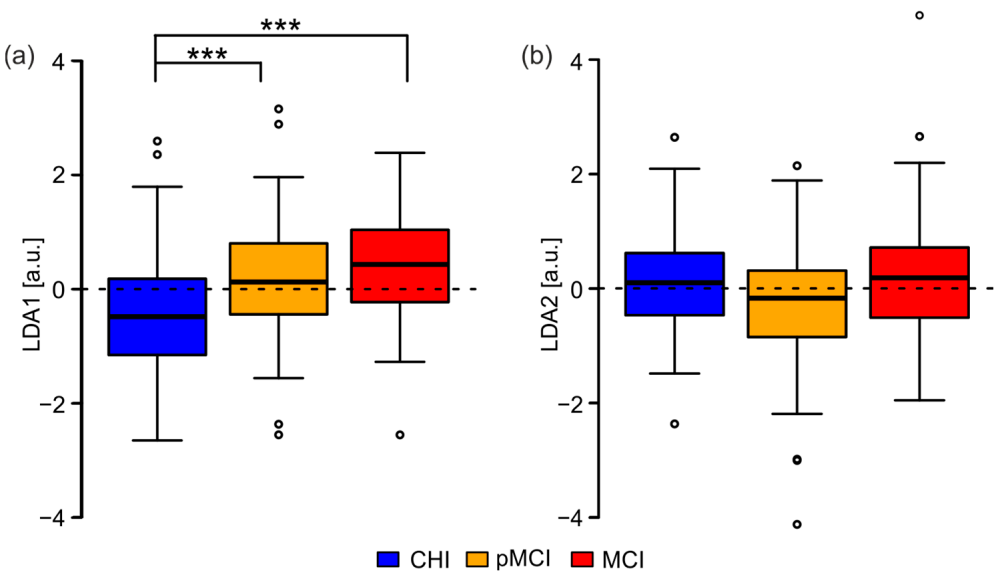

Figure 2. Boxplot of the LDA scales per group. (a) LDA1; (b) LDA2. CHI: cognitively healthy individuals; pMCI: participants with possible mild cognitive impairments; MCI: participants with mild cognitive impairments. ${ }^{+} p<0.1,{ }^{*} p<0.05,{ }^{* * *} p<0.001$.

Table 6. Confusion matrix of classification.

\begin{tabular}{|c|c|c|c|c|}
\hline & & \multicolumn{3}{|c|}{ Classification Based on Cognitive Assessments } \\
\hline & & $\mathrm{CHI}$ & pMCI & MCI \\
\hline 芯节 & $\begin{array}{c}\mathrm{CHI} \\
\mathrm{pMCI} \\
\mathrm{MCI}\end{array}$ & $\begin{array}{l}49 \\
20 \\
10\end{array}$ & $\begin{array}{l}26 \\
41 \\
13\end{array}$ & $\begin{array}{l}18 \\
24 \\
23\end{array}$ \\
\hline
\end{tabular}

(a) LDA1
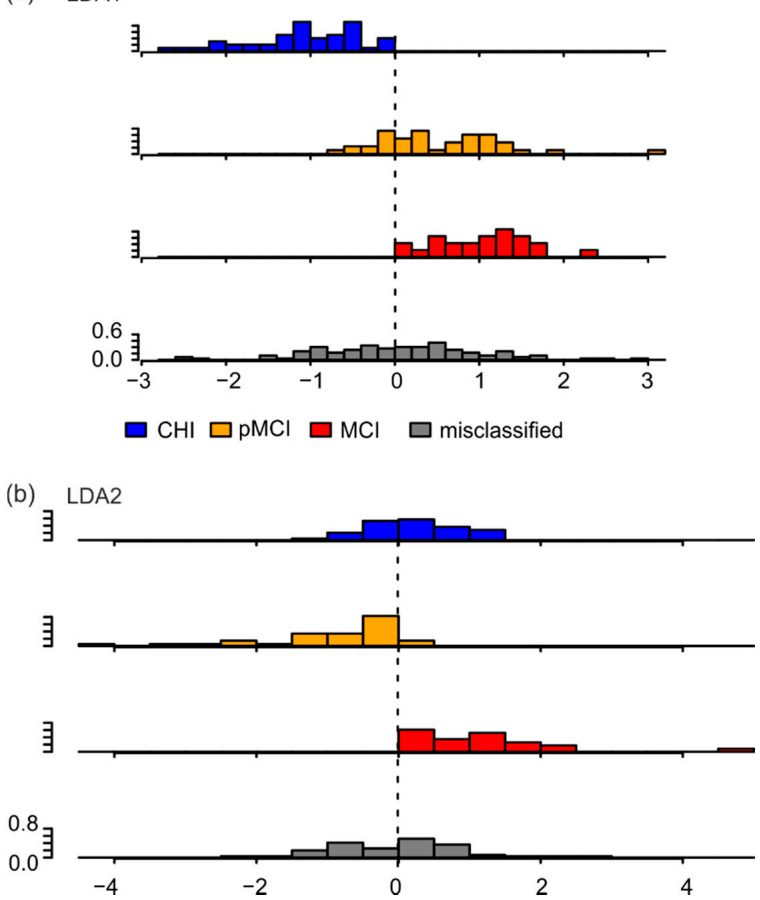

Figure 3. Histograms of probability densities of LDA values of correctly classified and misclassified participants. (a) LDA1; (b) LDA2. CHI: cognitively healthy individuals; pMCI: participants with possible mild cognitive impairments; MCI: participants with mild cognitive impairments. 
The LDA with only parameters significant to the effect group reclassified only $47 \%$ of participants into the correct class. The distribution of LDA scales for each group showed a significant difference $(p<0.05)$ between medians only for LDA1 between CHI and pMCI and between CHI and MCI (data not shown). The histograms of the probability densities of the LDA values of correctly classified and misclassified participants mainly show a broadening of the distribution of misclassified participants (Supplement Figure S1). Overall, this indicates that finger tapping behavior was conditioned by cognitive status in only a subset of participants.

\section{Discussion}

The aim of the study was to develop a system that uses tapping parameters in a self-selected and fast tapping mode to distinguish cognitively healthy individuals (CHI) from people with possible MCI ( $\mathrm{pMCI}$ ) and people with mild cognitive impairments (MCI), specifically for individuals over 80 years old. For this purpose, the finger tapping behavior of 225 subjects over 80 years old was analyzed. ANOVA revealed differences between groups (CHI, pMCI, MCI), sexes (male, female) and their interaction (group $\times$ sex) for the self-selected pace condition (four parameters) and for the fast pace condition (eight parameters). These parameters were used for classification by means of a linear discriminant analysis (LDA). The first LDA component showed significant differences between $\mathrm{CHI}$ and pMCI, CHI and MCI, and pMCI and MCI. Furthermore, the second LDA component showed significant differences between $\mathrm{CHI}$ and $\mathrm{pMCI}$ and between $\mathrm{pMCI}$ and MCI. Nevertheless, when the algorithm was used to classify individual participants, it was correct in only $50 \%$ of cases. This shows that tapping parameters were only partially useful for classification.

Our results showed that pMCI, a group first described in the SENDA study [10], differed from both $\mathrm{CHI}$ and MCI. Previous studies on tapping behavior were mainly conducted with Alzheimer's patients (e.g., [12]) or MCI patients, (e.g., the CDR. 5 group in [13]). In this study, we additionally showed that in the self-selected pace condition, not only participants with MCI but also those with pMCI had a significantly slower tapping rhythm and prolonged touch duration compared to CHI (Table 2: tap-cyle_median, tapduration_median, and plateau-duration_median).

However, the planned goal of classifying individual participants based on tapping parameters was only partially achieved. Thus, while 49 of $79 \mathrm{CHI}$ participants were correctly classified on the basis of their motor performance, 30 of these participants were classified as pMCI or MCI. Furthermore, 42 of $65 \mathrm{MCI}$ patients were apparently classified as $\mathrm{CHI}$ or pMCI. An explanation for the misclassification might be the simplicity of the task. Previous work has shown that no age effects exist in tasks with simple planned anticipatory grasp control, such as in tapping [29]; only tasks with higher complexity, such as activities of daily living, had recognizable differences [29]. In a recent study with a subset of the subjects described here, it was shown that all participants were comparably able to perform anticipatory grip strength control regardless of group membership [18]. It can therefore be assumed that the motor requirements of the tapping task were not sufficient to reliably separate between the groups.

In addition to the experimental condition, the neurological status of the participants must also be considered. All participants reported no neurological deficits (an exclusion criterion; see Methods). However, individuals may have had different degrees of age-related degeneration and in different relevant areas of the CNS (e.g., cortex, spinal cord, basal ganglia, cerebellum). Cortical activity can be measured via resting-state electroencephalography (EEG), usually performed with eyes closed and/or eyes open [30]. It is a measure of tonic brain activity [31] and this spontaneous EEG activity is thought to account for $80 \%$ of total brain activity $[30,32]$. Only a small additional percentage is accounted for by engagement in a task [32]. Thus, resting-state EEG studies describe the functional state of the cortex. A recent study [10] showed that in the subjects studied, cortical activity in 
resting-state EEG did not differ between groups. Therefore, group differences in tapping parameters cannot be derived from cortical differences between the groups.

For Parkinson's disease, as an example disease of the basal ganglia, it is known that patients show a faster tapping rhythm than healthy subjects [14]. In contrast, our data show that in the self-selected pace condition PMCI and MCI tapped significantly slower than $\mathrm{CHI}$, and in the fast pace condition there was a significant difference between $\mathrm{CHI}$ and MCI for this parameter (Table 2). This is consistent with behavior shown in MCI and Alzheimer's patients [12-14]. Therefore, it is reasonable to conclude that the group differences are not due to an influence of the basal ganglia.

The influence of spinal cord control can be derived from the parameter time-to-plateau (Figure 1). Tapping can be described as a diadochokinetic task. It consists of finger flexion followed by finger extension. The time to stop flexion and start extension is controlled by proprioceptive signals. The mean size of the parameter time-to-plateau gives information on the planned movement (the shorter the time to plateau, the faster the movement), and its variability gives information on the participant's proprioceptive control at the spinal cord level (the smaller the better). The parameter time-to-plateau was determined by the current speed of the movement (Figure 1, flexion) and the sensory feedback at touch, which could lead to deceleration of the movement and onset of the reverse movement (Figure 1, extension). If sensory feedback is insufficient, the stopping of the movement is delayed and much more variable. Thus, group differences can be inferred from the variability and mean magnitude of this parameter. For the self-selected pace condition, no group differences existed in either mean magnitude (Table 2 time-to-plateau_median) or variability (Table 2 time-to-plateau_iqr). In the fast pace condition, only one significant difference was found between CHI and pMCI or MCI for the parameter time-to-plateau_median. Therefore, in the fast pace condition, $\mathrm{CHI}$ performed a significantly faster motor program than the other groups. Because the variability of the time-to-plateau parameter was the same between groups in both pace conditions, it can be assumed that the degree of degeneration at the spinal cord level can be considered comparable between the groups.

The cerebellum is known to be generally important for coordinating motor performance, such as diadochokinesis, and it is additionally important for associating sensory information with movements as well as for adapting movements [33]. Some studies have highlighted the cerebellum's importance in the context of participants' associative learning of grip forces [34,35]. For example, in precision finger tasks such as the raspberry task $[36,37]$, half of the young participants showed a conditioned change in force at just the second presentation of the conditioning stimulus [35] and personal observation of DFK. In contrast, cerebellar patients were significantly worse than control subjects at learning the necessary association [34]. For a successful association between the conditioned stimulus and the motor action, participants needed a well-planned and controlled execution of the task [35]; in cerebellar patients, this execution was impaired [25]. It is therefore possible that restrictions in tapping behavior can be explained not only by cognitive impairments, but also by age-related decline of the cerebellum. This is accompanied by a reduced ability to associate sensory information with the necessary timing of tapping. As they are spatially separated from the regions related to manual motor performance, parts of the cerebellum are also correlated with cognitive performance [38-41]. The anterior lobe and the top of the superior posterior lobe are correlated with motor skills, and the bottom parts of the posterior superior lobe and the inferior lobe are correlated with cognition [39]. Degeneration of cerebellar regions associated with the somatomotor network is more pronounced than that of regions associated with dorsal attention, ventral attention, or frontoparietal networks [38]. Furthermore, age-related degeneration of the motor cerebellum is comparable to the degeneration found in cerebellar diseases [38]. In contrast, Alzheimer's patients show degeneration of the cognitive part of the cerebellum without concomitant increased degeneration in the motor cerebellum [41]. Notably, the cerebellum is generally considered to be resistant to the neurotoxic effects of soluble amyloid-beta $(A \beta)$, which is helpful in the early stages of AD [42]. However, assuming that a proportion of participants classified as 
$\mathrm{MCI}$ are in a precursor phase to AD, it is still reasonable to hypothesize that the influence of the cerebellum on tapping behavior should be considered an age-related limitation rather than an effect of the developing disease.

In conclusion, the 30 misclassified participants in the $\mathrm{CHI}$ group may have had more degeneration of the motor cerebellum than those correctly classified into the CHI group $(n=49)$. Indicators for this difference are the values for the tap-cycle (correctly classified CHI: $-0.697 \pm 0.008$; misclassified CHI: $-0.422 \pm 0.013$; mean \pm SEM in log [s]) and tapduration (correctly classified CHI: $-1.825 \pm 0.007$; misclassified CHI: $-1.448 \pm 0.016$; mean $\pm \mathrm{SEM}$ in $\log$ [s]) parameters in the self-selected condition. Thus, in this condition, the correctly classified CHI showed a significantly faster tapping rhythm with a shorter tapping duration ( $p<0.005$, Bonferroni corrected, both parameters). Similarly, it can be hypothesized that the 42 misclassified MCI participants had less degeneration of the cerebellum than the correctly classified MCI patients $(n=23)$. This can be seen from the values for the tap-cycle (correctly classified MCI: $-1,289 \pm 0.012$; misclassified MCI: $-1.407 \pm 0.005 ;$ mean \pm SEM in $\log [\mathrm{s}]$ ) and tap-duration (correctly classified MCI: $-2.017 \pm 0.012$; misclassified MCI: $-2.213 \pm 0.004$; mean \pm SEM in $\log [s])$ parameters in the fast pace condition. In this condition, the correctly classified MCI showed a slower tap rhythm $(p<0.1$, Bonferroni corrected) with a significantly longer tap duration $(p<0.004$, Bonferroni corrected).

Overall, when investigating whether cognitive state can be assessed based on simple finger movements (such as tapping), one must also consider the possible degeneration of relevant motor systems (e.g., the cerebellum). To establish tapping as a good classifier, researchers need to perform additional motor tests to specifically determine the degeneration of the aforementioned areas and adequately assess their impact on tapping behavior.

Supplementary Materials: The following are available online at https:/ / www.mdpi.com/article / 10.3390/jpm12020286/s1, Supplementary Table S1: Mean values of the tapping parameter for both conditions of each group (retransformed into physical dimensions). Supplementary Table S2: Logarithmized tapping parameter of female participants for both conditions and each group. Supplementary Table S3: Logarithmized tapping parameter of male participants for both conditions and each group. Supplementary Figure S1: Histograms of probability densities of LDA values of correctly classified and misclassified participants using only parameters that are significant for the effect group.

Author Contributions: S.F.: data collection; K.M.: project administration; J.R.: data revision, conceptualization of analysis; C.V.-R.: project conceptualization and administration, funding acquisition; S.F., K.M., J.R., C.V.-R. and D.F.K.: writing, review and editing; D.F.K., conceptualization, performance of the analysis, and writing of the first version of the manuscript. All authors contributed to manuscript revision and read and approved the submitted version. All authors have read and agreed to the published version of the manuscript.

Funding: This work was supported by the European Social Fund for Germany, the Sächsische Aufbaubank-Förderbank (SAB) of the Free State of Saxony (Grant No. 100310502), and Deutsche Forschungsgemeinschaft (DFG, German Research Foundation) under DFG Project-ID $416228727-$ SFB 1410). This funding source had no role in the study design, in the collection, analysis, and interpretation of data, or in the writing of the report. We acknowledge support from the Open Access Publication Fund of the University of Münster.

Institutional Review Board Statement: The studies involving human participants were reviewed and approved by Ethics Committee of the Chemnitz University of Technology, Faculty of Behavioral and Social Sciences (number V-232-17-KM-SENDA-07112017 approved on 19 December 2017).

Informed Consent Statement: The patients/participants provided written informed consent to participate in this study.

Data Availability Statement: The datasets analyzed during the current study are not publicly available but are available from the corresponding author on reasonable request. 


\begin{abstract}
Acknowledgments: The authors would like to thank all the participants for their contribution. Further thanks go to Brennecka from the Scientific Editorial Service of the University of Münster for proofreading the manuscript. We acknowledge support from the Open Access Publication Fund of the University of Muenster.
\end{abstract}

Conflicts of Interest: The authors declare no conflict of interest.

\title{
References
}

1. Müller, K.; Fröhlich, S.; Germano, A.M.C.; Kondragunta, J.; Agoitia Hurtado, M.F.d.C.; Rudisch, J.; Schmidt, D.; Hirtz, G.; Stollmann, P.; Voelcker-Rehage, C. Sensor-based systems for early detection of dementia (SENDA): A study protocol for a prospective cohort sequential study. BMC Neurol. 2020, 20, 84. [CrossRef] [PubMed]

2. Deutsche Alzheimer Gesellschaft e.V. Informationsblatt 1 Die Häufigkeit von Demenzerkrankungen. 2018. Available online: https:/ / www.deutsche-alzheimer.de/fileadmin/alz/pdf/factsheets/infoblatt1_haeufigkeit_demenzerkrankungen_dalzg.pdf (accessed on 25 October 2021).

3. Lin, P.-J.; Neumann, P.J. The economics of mild cognitive impairment. Alzheimers Dement. 2013, 9, 58-62. [CrossRef]

4. Frank, L.; Lenderking, W.R.; Howard, K.; Cantillon, M. Patient self-report for evaluating mild cognitive impairment and prodromal Alzheimer's disease. Alzheimers Res. Ther. 2011, 3, 12. [CrossRef] [PubMed]

5. Vogel, A.; Stokholm, J.; Gade, A.; Andersen, B.B.; Hejl, A.M.; Waldemar, G. Awareness of Deficits in Mild Cognitive Impairment and Alzheimer's Disease: Do MCI Patients Have Impaired Insight? Dement. Geriatr. Cogn. Disord. 2004, 17, 181-187. [CrossRef] [PubMed]

6. Farias, S.T.; Mungas, D.; Jagust, W. Degree of discrepancy between self and other-reported everyday functioning by cognitive status: Dementia, mild cognitive impairment, and healthy elders. Int. J. Geriatr. Psychiatry 2005, 20, 827-834. [CrossRef]

7. Argüelles, S.; Loewenstein, D.A.; Eisdorfer, C.; Argüelles, T. Caregivers' Judgments of the Functional Abilities of the Alzheimer's Disease Patient: Impact of Caregivers' Depression and Perceived Burden. J. Geriatr. Psychiatry Neurol. 2001, 14, 91-98. [CrossRef]

8. Nasreddine, Z.S.; Phillips, N.A.; Bédirian, V.; Charbonneau, S.; Whitehead, V.; Collin, I.; Cummings, J.L.; Chertkow, H. The Montreal Cognitive Assessment, MoCA: A Brief Screening Tool For Mild Cognitive Impairment. J. Am. Geriatr. Soc. 2005, 53, 695-699. [CrossRef]

9. Moms, J.C.; Heyman, A.; Mohs, R.C.; Hughes, J.P.; van Belle, G.; Fillenbaum, G.; Mellits, E.D.; Clark, C. The Consortium to Establish a Registry for Alzheimer's Disease (CERAD). Part I. Clinical and neuropsychological assesment of Alzheimer's disease. Neurology 1989, 39, 1159. [CrossRef]

10. Fröhlich, S.; Kutz, D.F.; Müller, K.; Voelcker-Rehage, C. Characteristics of Resting State EEG Power in 80+-Year-Olds of Different Cognitive Status. Front. Aging Neurosci. 2021, 13, 469. [CrossRef]

11. Fröhlich, S.; Müller, K.; Voelcker-Rehage, C. Normative Data for the CERAD-NP for Healthy High-Agers (80-84 years) and Effects of Age-Typical Visual Impairment and Hearing Loss. J. Int. Neuropsychol. Soc. 2021, in press. [CrossRef]

12. Rabinowitz, I.; Lavner, Y. Association between Finger Tapping, Attention, Memory, and Cognitive Diagnosis in Elderly Patients. Percept. Mot. Ski. 2014, 119, 259-278. [CrossRef] [PubMed]

13. Bangert, A.S.; Balota, D.A. Keep Up the Pace: Declines in Simple Repetitive Timing Differentiate Healthy Aging from the Earliest Stages of Alzheimer's Disease. J. Int. Neuropsychol. Soc. 2012, 18, 1052-1063. [CrossRef] [PubMed]

14. Roalf, D.R.; Rupert, P.; Mechanic-Hamilton, D.; Brennan, L.; Duda, J.E.; Weintraub, D.; Trojanowski, J.Q.; Wolk, D.; Moberg, P.J. Quantitative assessment of finger tapping characteristics in mild cognitive impairment, Alzheimer's disease, and Parkinson's disease. J. Neurol. 2018, 265, 1365-1375. [CrossRef]

15. Cole, K.J.; Rotella, D.L.; Harper, J.G. Mechanisms for Age-Related Changes of Fingertip Forces during Precision Gripping and Lifting in Adults. J. Neurosci. 1999, 19, 3238-3247. [CrossRef] [PubMed]

16. Johansson, R.S.; Cole, K.J. Sensory-motor coordination during grasping and manipulative actions. Curr. Opin. Neurobiol. 1992, 2, 815-823. [CrossRef]

17. Johansson, R.S.; Cole, K.J. Grasp stability during manipulative actions. Can. J. Physiol. Pharmacol. 1994, 72, 511-524. [CrossRef] [PubMed]

18. Rudisch, J.; Müller, K.; Kutz, D.F.; Brich, L.; Sleimen-Malkoun, R.; Voelcker-Rehage, C. How Age, Cognitive Function and Gender Affect Bimanual Force Control. Front. Physiol. 2020, 11, 245. [CrossRef]

19. Breton, A.; Casey, D.; Arnaoutoglou, N.A. Cognitive tests for the detection of mild cognitive impairment (MCI), the prodromal stage of dementia: Meta-analysis of diagnostic accuracy studies. Int. J. Geriatr. Psychiatry 2019, 34, 233-242. [CrossRef]

20. Albert, M.S.; DeKosky, S.T.; Dickson, D.; Dubois, B.; Feldman, H.H.; Fox, N.C.; Gamst, A.; Holtzman, D.M.; Jagust, W.J.; Petersen, R.C.; et al. The diagnosis of mild cognitive impairment due to Alzheimer's disease: Recommendations from the National Institute on Aging-Alzheimer's Association workgroups on diagnostic guidelines for Alzheimer's disease. Alzheimers Dement. 2011, 7, 270-279. [CrossRef]

21. Petersen, R.C.; Caracciolo, B.; Brayne, C.; Gauthier, S.; Jelic, V.; Fratiglioni, L. Mild cognitive impairment: A concept in evolution. J. Intern. Med. 2014, 275, 214-228. [CrossRef] 
22. Petersen, R.C.; Lopez, O.; Armstrong, M.J.; Getchius, T.S.D.; Ganguli, M.; Gloss, D.; Gronseth, G.S.; Marson, D.; Pringsheim, T.; Day, G.S.; et al. Practice guideline update summary: Mild cognitive impairment. Rep. Guidel. Dev. Dissem. Implement. Subcomm. Am. Acad. Neurol. 2018, 90, 126-135. [CrossRef] [PubMed]

23. Yan, J.H. Effects of Aging on Linear and Curvilinear Aiming Arm Movements. Exp. Aging Res. 2000, 26, 393-407. [CrossRef] [PubMed]

24. R-CoreTeam. R: A Language and Environment for Statistical Computing; R Foundation for Statistical Computing: Vienna, Austria, 2018.

25. Meindl, T.; Schmid, B.C.; Timmann, D.; Kolb, F.P.; Kutz, D.F. Contribution of the Cerebellum to the Coupling of Grip Force and Pull Force During an Isometric Precision Grip Task. Cerebellum 2012, 11, 167-180. [CrossRef] [PubMed]

26. Lawrence, M.A. ez: Easy Analysis and Visualization of Factorial Experiments, R package version 4.4-0; R Core Team: Vienna, Austria, 2016.

27. Bakeman, R. Recommended effect size statistics for repeated measures designs. Behav. Res. Methods 2005, 37, 379-384. [CrossRef]

28. Venables, W.N.; Ripley, B.D. Modern Applied Statistics with S, 4th ed.; Springer: New York, BY, USA, $2002 ;$ p. 498.

29. Diermayr, G.; McIsaac, T.L.; Gordon, A.M. Finger Force Coordination Underlying Object Manipulation in the Elderly-A Mini-Review. Gerontology 2011, 57, 217-227. [CrossRef]

30. Cave, A.E.; Barry, R.J. Sex differences in resting EEG in healthy young adults. Int. J. Psychophysiol. 2021, 161, 35-43. [CrossRef]

31. Klimesch, W. EEG alpha and theta oscillations reflect cognitive and memory performance: A review and analysis. Brain Res. Rev. 1999, 29, 169-195. [CrossRef]

32. Shulman, R.G.; Rothman, D.L.; Behar, K.L.; Hyder, F. Energetic basis of brain activity: Implications for neuroimaging. Trends Neurosci. 2004, 27, 489-495. [CrossRef]

33. Manto, M.; Bower, J.M.; Conforto, A.B.; Delgado-Garcia, J.M.; da Guarda, S.N.; Gerwig, M.; Habas, C.; Hagura, N.; Ivry, R.B.; Marien, P.; et al. Consensus paper: Roles of the cerebellum in motor control-the diversity of ideas on cerebellar involvement in movement. Cerebellum 2012, 11, 457-487. [CrossRef]

34. Kutz, D.F.; Schmid, B.C.; Meindl, T.; Timmann, D.; Kolb, F.P. Contribution of the Cerebellum in Cue-Dependent Force Changes During an Isometric Precision Grip Task. Cerebellum 2016, 15, 439-450. [CrossRef]

35. Schmid, B.C.; Meindl, T.; Timmann, D.; Kolb, F.P.; Kutz, D.F. Motor learning of cue-dependent pull-force changes during an isometric precision grip task. Hum. Mov. Sci. 2015, 39, 138-153. [CrossRef] [PubMed]

36. Kutz, D.F.; Wölfel, A.; Meindl, T.; Timmann, D.; Kolb, F.P. Spatio-temporal human grip force analysis via sensor arrays. Sensors 2009, 9, 6330-6345. [CrossRef] [PubMed]

37. Kutz, D.F.; Wölfel, A.; Timmann, D.; Kolb, F.P. Dynamic torque during a precision grip task comparable to picking a raspberry. J. Neurosci. Methods 2009, 177, 80-86. [CrossRef] [PubMed]

38. Hulst, T.; van der Geest, J.N.; Thürling, M.; Goericke, S.; Frens, M.A.; Timmann, D.; Donchin, O. Ageing shows a pattern of cerebellar degeneration analogous, but not equal, to that in patients suffering from cerebellar degenerative disease. NeuroImage 2015, 116, 196-206. [CrossRef] [PubMed]

39. Koppelmans, V.; Hoogendam, Y.Y.; Hirsiger, S.; Mérillat, S.; Jäncke, L.; Seidler, R.D. Regional cerebellar volumetric correlates of manual motor and cognitive function. Brain Struct. Funct. 2017, 222, 1929-1944. [CrossRef] [PubMed]

40. Koziol, L.F.; Budding, D.; Andreasen, N.; D’Arrigo, S.; Bulgheroni, S.; Imamizu, H.; Ito, M.; Manto, M.; Marvel, C.; Parker, K.; et al. Consensus Paper: The Cerebellum's Role in Movement and Cognition. Cerebellum 2014, 13, 151-177. [CrossRef]

41. Gellersen, H.M.; Guell, X.; Sami, S. Differential vulnerability of the cerebellum in healthy ageing and Alzheimer's disease. NeuroImage Clin. 2021, 30, 102605. [CrossRef]

42. Liang, K.J.; Carlson, E.S. Resistance, vulnerability and resilience: A review of the cognitive cerebellum in aging and neurodegenerative diseases. Neurobiol. Learn. Mem. 2020, 170, 15. [CrossRef] 